\title{
Fluctuation conductivity in two-band superconductor $\mathrm{SmFeAsO}{ }_{0.8} \mathbf{F}_{0.2}$
}

\author{
I.N. ASKeRZADE ${ }^{1,2 *}$, R.T. TAgIYEVA ASKeRBEYLI ${ }^{2,3}$ \\ ${ }^{1}$ Computer Engineering Department and Center of Excellence of Superconductivity, Ankara University, \\ Ankara, 06100, Turkey \\ ${ }^{2}$ Institute of Physics Azerbaijan National Academy of Sciences, Baku, Az1143, Azerbaijan \\ ${ }^{3}$ Business Administration Department of Karabuk University, Demircelik Campus, Karabuk, Turkey
}

\begin{abstract}
In this study we have calculated the fluctuation conductivity near critical temperature of $\mathrm{SmFe}_{\mathrm{AsO}} \mathrm{O}_{0.8} \mathrm{~F}_{0.2}$ superconductor using two-band Ginzburg-Landau theory. It was illustrated that in the absence of external magnetic field, the two-band model reduced to a single effective band theory with modified temperature dependences. The calculations revealed three-dimensional character of fluctuations of conductivity in the new Fe-based superconductor $\mathrm{SmFeAsO}_{0.8} \mathrm{~F}_{0.2}$. It has been shown that such a model is in good agreement with experimental data for this compound.
\end{abstract}

Keywords: fluctuation; conductivity; Ginzburg-Landau theory; two-band superconductivity

(C) Wroclaw University of Technology.

\section{Introduction}

Soon after the discovery of superconductivity in Fe-based compounds [1], they have been indicated as the new unconventional superconductors which could compete with high- $\mathrm{T}_{\mathrm{c}}$ cuprates for application purposes. For Fe-based materials, several common features have been identified. In general, superconductivity appears upon chemical doping of an antiferromagnetic parent compound with an optimal doping level, which yields the largest transition temperature; the parent compound is an almost compensated semimetal, whose Fermi surface fulfills the nesting condition; the presence of Fe which used to be considered detrimental for superconductivity may be a crucial ingredient therein; instead and finally spin fluctuation mediated pairing has been suggested [2].

Among various $\mathrm{Fe}$ based superconductors $\mathrm{SmFeAsO}{ }_{1-\mathrm{x}} \mathrm{F}_{\mathrm{x}}$ stands out as a unique material, with a critical temperature about $55 \mathrm{~K}$ [3]. The highest temperature of doped $\mathrm{SmFeAsO}_{1-\mathrm{x}} \mathrm{F}_{\mathrm{x}}$ systems is $55 \mathrm{~K}$ for $\mathrm{x}=0.2[4,5]$. The Fermi surface

*E-mail: imasker@eng.ankara.edu.tr of $\mathrm{SmFeAsO}{ }_{1-\mathrm{x}} \mathrm{F}_{\mathrm{x}}$ is formed by three concentric hole cylinders at the $\Gamma$ point of Brilloun zone and a small hole cylinder near the zone boundary $[6,7]$. Many-band character of superconducting state of $\mathrm{SmFeAsO}_{1-\mathrm{x}} \mathrm{F}_{\mathrm{x}}$ was confirmed by ARPES [8] and de Haas-van Alphen experiments [9].

The main peculiarity of superconductivity in Fe-based compounds is related to the multiband character of state $[4,6,7]$. It is useful to note that the multiband Bardeen-Cooper-Schrieffer theory for Fe-based superconductors was suggested in recent studies $[10,11]$. The multiband Eliashberg theory with antiferromagnetic spin fluctuations was considered in [12]. The four-band Eliashberg model was applied for the study of thermodynamic properties of Fe-based compounds in paper [13]. Recently, the fluctuation conductivity of $\mathrm{SmFeAsO}_{1-\mathrm{x}} \mathrm{F}_{\mathrm{x}}$ has been measured in study [14]. They found that the fluctuation conductivity cannot be described by the standard Ginzburg-Landau theory with a single isotropic energy band. In the present work, the fluctuation conductivity of $\mathrm{SmFeAsO}{ }_{1-\mathrm{x}} \mathrm{F}_{\mathrm{X}}$ was calculated using the two-band Ginzburg-Landau theory. Two-band Ginzburg-Landau theory was developed 
in [15-19]. Obtained equations were applied for the study of different physical properties of $\mathrm{MgB}_{2}$, nonmagnetic borocarbides and good agreement with experimental data was achieved. Very recently similar theory has been applied for the estimation of specific heat jump in new Fe-based superconductor $\mathrm{KFe}_{2} \mathrm{As}_{2}$ [20]. Fitting parameters for calculation of fluctuation conductivity were taken from the microscopic theory for $\mathrm{SmFeAsO}_{1-\mathrm{x}} \mathrm{F}_{\mathrm{x}}$ compound. Finally, the results of calculations were compared with the experimental data [14].

\section{Basic equations}

The fluctuations of the order parameter above a critical temperature can also contribute to the electrical conductivity, which is also called as paraconductivity [21]. Average current in the absence of external magnetic field, using Ginzburg-Landau theory, can be written as [22]:

$$
j(\vec{r})=-i \frac{e \hbar}{2 m}\left(\Psi^{*} \nabla \Psi-\Psi \nabla \Psi^{*}\right)
$$

Using wave decomposition of order parameter $\Psi(\overrightarrow{\mathrm{r}})$ as $\Psi(\overrightarrow{\mathrm{r}})=\sum_{\overrightarrow{\mathrm{q}}} \mathrm{c}_{\overrightarrow{\mathrm{q}}} \psi_{\overrightarrow{\mathrm{q}}}(\overrightarrow{\mathrm{r}})$ in equation 1 [22], we can get:

$$
j(\vec{q})=\frac{2 e \hbar V}{2 m} \sum_{\vec{q}}(2 \vec{p}+\vec{q}) \psi_{\vec{p}}^{*} \psi_{\vec{p}+\vec{q}}
$$

For the calculation of electrical conductivity $\sigma$ we will use Kubo expression [23]:

$$
\sigma(\vec{q})=\frac{1}{2 T} \int_{-\infty}^{\infty} d t\left\langle j_{\vec{q}}(t) j_{\vec{q}}(0)\right\rangle
$$

In equation 3 , notation $\langle\ldots\rangle$ means statistical averaging procedure. Substituting (2) into (1), taking into account the presented wave decomposition of order parameter $\Psi(\overrightarrow{\mathrm{r}})$ and limiting ourselves to $\mathrm{q}=0$, we can get following expression:

$\sigma_{i j}(0)=\frac{(2 e)^{2} \hbar^{2} V^{2}}{32 m^{2} T} \int_{-\infty}^{\infty} d t \sum_{\vec{k}} \vec{k}_{i} \vec{k}_{j}\left|\left\langle\psi_{k}^{*}(t) \psi_{k}(0)\right\rangle\right|^{2}$

For the calculation of correlation function $\left\langle\psi_{\mathrm{k}}^{*}(\mathrm{t}) \psi_{\mathrm{k}}(0)\right\rangle$, the time-dependent single-band
Ginzburg-Landau equation was used in [21]. For single-band superconductors, the solution of Ginzburg-Landau equation has a form:

$$
\psi_{k}(t)=\psi_{k}(0) \exp \left(-\frac{t}{\tau_{k}}\right)
$$

with the relaxation parameter $\Gamma$ and relaxation time $\tau_{\mathrm{k}}$ :

$$
\tau_{k}=\frac{4 m}{\Gamma \hbar^{2}} \frac{1}{k^{2}+\xi^{-2}}
$$

where $\xi$ is the coherence length of superconductor. Calculations of integrals equations 3 and 4 lead to the classical Aslamazov-Larkin result [21]:

$$
\Delta \sigma=\left\{\begin{array}{lll}
\frac{1}{8 \pi}\left(\frac{e}{\hbar}\right)^{2}\left(\frac{\alpha m T_{c}}{T_{c}-T}\right)^{\frac{1}{2}}, & 3 d & \text { case } \\
\frac{1}{16} \frac{e^{2}}{\hbar d} \frac{T_{c}}{T_{c}-T}, & 2 d \quad \text { case }
\end{array}\right.
$$

As mentioned before, the fluctuation conductivity in $\mathrm{SmFeAsO}_{1-\mathrm{x}} \mathrm{F}_{\mathrm{x}}$ superconductor cannot be described by a single-band Ginzburg-Landau theory. We will adapt the two-band Ginzburg-Landau theory for this purpose. Free energy functional for two-band superconductors has the following form [15-19]:

$$
F_{S C}=\int d^{3} r\left(F_{1}+F_{12}+F_{2}+H^{2} / 8 \pi\right)
$$

where

$$
\begin{aligned}
& F_{i}= \frac{\hbar^{2}}{4 m_{i}}\left|\left(\nabla-\frac{2 \pi i \vec{A}}{\Phi_{0}}\right) \Psi_{i}\right|^{2}+\alpha_{i}(T)\left|\Psi_{i}\right|^{2} \\
&+\beta_{i}\left|\Psi_{i}\right|^{4} / 2 \\
& F_{12}= \varepsilon\left(\Psi_{1}^{*} \Psi_{2}+\text { c.c. }\right) \\
&+\varepsilon_{1}\left\{\left(\nabla+\frac{2 \pi i \vec{A}}{\Phi_{0}}\right) \Psi_{1}^{*}\left(\nabla-\frac{2 \pi i \vec{A}}{\Phi_{0}}\right) \Psi_{2}+\text { c.c. }\right\}
\end{aligned}
$$

$\mathrm{m}_{\mathrm{i}}$ are the masses of electrons belonging to different bands $(i=1,2) ; \alpha_{i}=\gamma_{i}\left(T-T_{c i}\right)$ are the quantities linearly dependent on temperature $\mathrm{T}, \beta_{\mathrm{i}}$ and $\gamma_{i}$ are constant coefficients, $\varepsilon$ and $\varepsilon_{1}$ describe the 
interaction between order parameters and their gradients of different bands, respectively, $\mathrm{H}$ is the external magnetic field and $\Phi_{0}$ is the magnetic flux quantum. In equations 8,9 and 10 , the order parameters $\Psi_{1,2}$ are assumed to be slowly varying in space. Minimization procedure of the free-energy functional 8 yields the Ginzburg-Landau equations, describing the two-band superconductors:

$$
\begin{aligned}
& -\frac{\hbar^{2}}{4 m_{1}}\left(\frac{d^{2}}{d x^{2}}-\frac{x^{2}}{l_{s}^{4}}\right) \Psi_{1}+\alpha_{1}(T) \Psi_{1}+\varepsilon \Psi_{2} \\
& +\varepsilon_{1}\left(\frac{d^{2}}{d x^{2}}-\frac{x^{2}}{l_{s}^{2}}\right) \Psi_{2}+\beta_{1}\left|\Psi_{1}\right|^{2} \Psi_{1}=0 \\
& -\frac{\hbar^{2}}{4 m_{2}}\left(\frac{d^{2}}{d x^{2}}-\frac{x^{2}}{l_{s}^{4}}\right) \Psi_{2}+\alpha_{2}(T) \Psi_{2}+\varepsilon \Psi_{1} \\
& +\varepsilon_{1}\left(\frac{d^{2}}{d x^{2}}-\frac{x^{2}}{l_{s}^{2}}\right) \Psi_{1}+\beta_{2}\left|\Psi_{2}\right|^{2} \Psi_{2}=0
\end{aligned}
$$

where $1_{\mathrm{s}}^{-2}=\frac{\hbar \mathrm{c}}{2 \mathrm{eH}}$ is so-called magnetic length. In the general case, the signs of the parameters of interband interaction $\varepsilon$ and $\varepsilon_{1}$ in equations 11 and 12 can be arbitrary. As shown in [18], in the absence of external magnetic field, the order parameters $\Psi_{1,2}$ are proportional to each other with temperature dependent coefficient C: $\Psi_{1}=C \Psi_{2}=-\frac{\varepsilon}{\alpha_{1}(\mathrm{~T})} \Psi_{2}$. In this approach, the system of Ginzburg-Landau equations reduces to a single equation:

$$
\begin{gathered}
-\frac{\hbar^{2}}{4 m^{*}}\left(\frac{d^{2}}{d x^{2}}-\frac{x^{2}}{l_{s}^{2}}\right) \Psi_{1}(x)+\alpha^{*}(T) \Psi_{1}+ \\
+\beta^{*} \Psi_{1}^{3}=0
\end{gathered}
$$

in which the coefficients are modified as:

$$
\begin{gathered}
m^{*}=\frac{m}{1-\frac{4 m_{1} \varepsilon_{1}}{C \hbar^{2}}}, \alpha^{*}(T)=\left(\alpha_{1}(T) \alpha_{2}(T)-\varepsilon^{2}\right), \\
\beta^{*}=\beta_{1}
\end{gathered}
$$

Very recently the equations 13 and 14 for effective single band Ginzburg-Landau approach were used to calculate the fluctuation part of diamagnetism in two-band superconductors [24] and a good agreement with experimental data was achieved.

\section{Results and discussion}

The above developed effective single band approach for two-band Ginzburg-Landau theory has been applied for the calculation of fluctuation conductivity in $\mathrm{SmFeAsO}{ }_{1-\mathrm{x}} \mathrm{F}_{\mathrm{x}}$ compound. The results of the calculations, obtained by using Aslamazov-Larkin expression (7) with modified parameters for temperature dependence (14) are presented in the Fig. 1. For comparison of the calculations, the experimental data of the fluctuation conductivity in $\mathrm{SmFeAsO}_{1-\mathrm{x}} \mathrm{F}_{\mathrm{X}}$ [14] are also presented in this figure. For the theoretical calculations the following fitting parameters were used: $\mathrm{T}_{\mathrm{c}}=$ $55 \mathrm{~K}, \mathrm{~T}_{\mathrm{c} 1} \approx 12.5 \mathrm{~K}, \mathrm{~T}_{\mathrm{c} 2} \approx 26.8 \mathrm{~K}, \frac{\varepsilon^{2}}{\gamma_{1} \gamma_{2} \mathrm{~T}_{\mathrm{c}}^{2}} \approx 0.399$, $\eta=\frac{\mathrm{T}_{\mathrm{c}} \mathrm{m}_{2} \varepsilon_{1} \gamma_{2}}{\hbar^{2} \varepsilon} \approx-0.021, \mathrm{r}_{\mathrm{m}}=\frac{\gamma_{1} \mathrm{~m}_{1}}{\gamma_{2} \mathrm{~m}_{2}} \approx 3.2$. In choosing of these parameters we have taken into account results of experimental works $[25,26]$ and results of other microscopic calculations for Sm compound $[4,5]$. Experimental results for fluctuation conductivity in $\mathrm{SmFeAsO}_{1-\mathrm{x}} \mathrm{F}_{\mathrm{x}}$ presented in Fig. 1 by full squares were taken from the study [14]. Results of calculations using two-band GinzburgLandau theory reduced to the effective single-band case, are shown by triangles in Fig. 1.

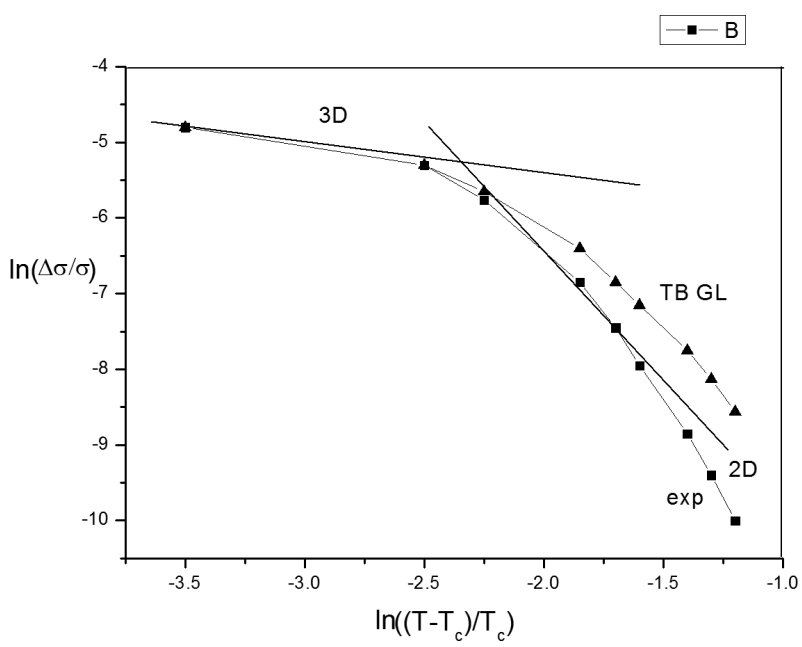

Fig. 1. Three dimensional character of fluctuation conductivity in $\mathrm{SmFeAsO}_{1-\mathrm{x}} \mathrm{F}_{\mathrm{x}}$. Full squares correspond to experimental data [14], triangles to two-band Ginzburg-Landau equations, straight lines to Aslamazov-Larkin expressions.

For a comparative analysis, the results obtained in an effective single band approximation for various temperature regimes are also presented by straight lines (Fig. 1). As can be seen from the figure, using equation 14, derived with allowance 
for the two band character of superconductors, provides a better agreement with the experimental data for $\mathrm{SmFeAsO}_{1-\mathrm{x}} \mathrm{F}_{\mathrm{x}}$. In the case of a single band Ginzburg-Landau theory, the AslamazovLarkin expression (7) for the fluctuation conductivity has the following structure: $\left(T_{c}-T\right)^{-v}$, where $v$ is a parameter that takes various values in different regimes (e.g. $v=0.5$ for three dimensional case and $v=1$ for the two dimensional case). In Fig. 1, this behavior corresponds to the straight lines with different slopes. An analysis of the data in the Fig. 1 shows that equation 14 obtained in this study, using the two-band model, provides a better description of the experimental points than the single band approximation does.

Finally, let us discuss the anomalous MakiThompson contribution in paraconductivity in $\mathrm{SmFeAsO}_{1-\mathrm{X}} \mathrm{F}_{\mathrm{X}}$ compound. This anomalous term has the same singularity in $\mathrm{T}_{\mathrm{c}}-\mathrm{T}$ as in Aslamazov-Larkin one [27, 28]. Maki-Thompson contribution is related to Andreev reflection by fluctuations Cooper pair and does not appear in usual time-dependent Ginzburg-Landau theory [29]. In the study [27] Maki found that in threedimensional case, fluctuation conductivity is four times larger than the Aslamazov-Larkin one (7). Maki-Thompson contribution has a purely quantum nature and was calculated using the diagram technique [27-29]. As shown in studies [30, 31] this contribution turns out to be strongly phase sensitive and non-s-wave suppresses the anomaly of Maki-Thompson process. It is generally accepted that the symmetry of order parameter in $\mathrm{Fe}$ based superconductors is different from swave pairing [4-6]. Our consideration developed above is applicable for three-dimensional superconductors. The second moment is related with that the pair-breaking in Fe-based compounds is weak [32]. In [32] it was shown extremely small character of the phase pair-breaking time in $\mathrm{SrFe}_{2} \mathrm{As}_{2}$ compound, which led to neglecting of Maki-Thompson contribution in this superconductor. This conclusion suggests a possible non-conventional superconductivity in the $\mathrm{Fe}$ based materials as it happens in high-temperature superconductors.
As a result, it was shown in this study that fluctuations in the new superconductor $\mathrm{SmFeAsO}_{1-\mathrm{X}} \mathrm{F}_{\mathrm{X}}$ reveal three dimensional character and can be described using two-band GinzburgLandau equations. This theory is reduced to an effective single-band approach and gives good agreement with experimental data [14]. It is useful to note that the calculations developed in this paper can be adopted to other Fe-based many-band superconductors after a proper choosing of fitting parameters.

\section{Acknowledgements}

This study was supported by TUBITAK Research Project $110 \mathrm{~T} 748$.

\section{References}

[1] Kamihara Y., Watanabe T., Hirano M., Hosono H., J. Am. Chem. Soc., 130 (2008), 3296.

[2] Mazin I.I., Singh D.J., Johannes M.D., DU M.H., Phys. Rev. Lett., 101 (2008), 057003.

[3] Wang C., Li C., L., Chi S., Zhu Z., Ren Z., Li Y. Wang Y., Lin X., Luo Y., Jiang S., Xu X., GaO G., XU Z., EPL-Europhys. Lett., 83 (2008), 67006.

[4] ReN Z.A., ZhaO Z.X., Adv. Mater., 21 (2009), 4584.

[5] Sadovskit S.V., Phys. USP.+, 178 (2008), 1243.

[6] ASKERZADE I., Unconventional superconductors. anisotropy and multiband effects, Springer-Verlag, New York, 2012, p. 177.

[7] Shein I.R., IVAnovskiI A.L., Solid State Commun., 150 (2010), 152.

[8] Philip J.W., Puzniak M.R., Balakirev F., RoGACKI K., KARPINSKI J., ZHIGADLO N.D., BARTLOGG B., Nat. Mater., 10 (8) (2010), 628.

[9] Tropeano M., Pallecchi I., Cimberle M.R., Ferdeghini C., Lamura G., Vignolo M., Martinelli A., Palenzona A., Putti M., Supercond. Sci. Tech., 23 (2010), 054001.

[10] BARZYKIN V., GORKOV L.P., JETP Lett.+, 88 (2008), 131.

[11] Dolgov O.V., Mazin I.I., Parker D., Phys. Rev. B, 79 (2008), 060502.

[12] Ummarino G.A., Phys. Rev. B, 83 (2011), 092508.

[13] Benfato L., Cappelluti E., Castelliani C., Phys. Rev. B, 80 (2009), 214522.

[14] Fanfarillo L., Benfato L., Caprara S., Phys. Rev. B, 79 (2009), 172508

[15] Askerzade I.N., Tech. Phys.+, 52 (2009), 977.

[16] Askerzade I.N., Tanatar B., Mod. Phys. Lett. B, 17 (2003), 11

[17] ASKERZADE I.N., JETP Lett.+, 81 (2005), 583.

[18] Askerzade I.N., Tech. Phys. +, 55 (2010), 896.

[19] Askerzade I.N., J. Korean Phys. Soc., 45 (2) (2004), 475 
[20] Askerzade I.N., Mater. Sci.-Poland, 45 (2) (2014), [27] MaKi K., Prog. Theor. Phys., 39 (1968), 897. 465.

[28] Thompson R.S., Phys. Rev. B, 1 (1970), 327.

[21] Aslamazov L.G., Larkin A.I., Sov. Phys. Solid State, 10 (1968), 875.

[22] Abrikosov A.A., Fundamentals theory of metals, North Holland, Amsterdam, 1988, p. 680.

[29] LARKIN A., VARLAMOV A.A., Theory of fluctuations of superconductors, Oxford Scientific Publishing, Oxford, 2005, p. 418.

[23] Landau L.D., Lifshitz E.M., Statistical Physics, Vol. 9, Pergamon Pres, Oxford, 1980.

[30] YIP S., Phys. Rev. B, 41 (1990), 2612.

[31] Carretta P., Rigamonti A., VArlamov A.A., LIVANOV D.V., Phys. Rev. B, 54 (1996), R9682.

[24] Askerzade I.N., J. Supercond. Nov. Magn., 28 (2) (2015), 319.

[25] Zhigadlo N.D., Katrych S., Kukovski Z.K., J. Phys.-Condens. Mat., 20 (2008), 342202.

[26] Jaroszynski J., Scott C., Riggs F., Hunte F., Phys. Rev. B, 78 (2008), 064611.

Received 2014-12-01

Accepted 2015-03-18 